\title{
Comparison of Marie Stopes scalpel and electrocautery no-scalpel vasectomy techniques
}

\author{
Timothy Black, MRCP, MFF, Chief Executive, Marie Stopes International, London, UK; Colin Francome, MA, PhD, Emeritus \\ Professor of the Sociology of Health, Middlesex University, London, UK and Editor of the Middlesex University Press Health \\ Series
}

Correspondence: Dr T Black, Chief Executive, Marie Stopes International, 153-157 Cleveland Street, London W1T 6QW, UK.Fax:+44 (0) 1403 891523.E-mail: timblack@stopes.org.uk

(Accepted 16th January 2003)

Journal of Family Planning and Reproductive Health Care 2003; 29(2): 32-34

\begin{abstract}
Objective. To compare the intra-operative experience and postoperative sequelae between the standard Marie Stopes scalpel vasectomy procedure and electrocautery nonscalpel vasectomy (ENSV) techniques.

Design. Randomised prospective comparative study.

Setting. Marie Stopes vasectomy centres in the UK.

Participants. A total of 325 men undergoing vasectomy between January and June 1999.

Intervention. Random allocation to the two study arms plus questionnaires at 4 and 14 weeks postoperatively.

Main outcome measures. Ease and speed of the procedure; pain levels during and after the procedure; early postoperative complications and time taken to return to work and sexual activity.

Results. The ENSV technique was marginally quicker to perform. Pain levels intra-operatively were comparable. Response rates to the questionnaire were $84.6 \%$ and $37 \%$ at 4 and 14 weeks, respectively. The ENSV group experienced less pain and bleeding from the wound postoperatively and were quicker to heal. For men who experienced postoperative problems, the time taken to return to work was marginally better in the ENSV group. The time taken to return to sexual activity was marginally faster in the ENSV group.

Conclusion. The ENSV procedure appears to be suitable for mass application in locations where electricity is available.
\end{abstract}

\section{Key message point}

- The electrocautery no-scalpel technique of exposing the vasa was virtually bloodless, relatively painless and without adverse complications.

\section{Introduction}

The study was a prospective comparison of the standard Stopes procedure using a scalpel and a no-scalpel vasectomy technique using electrocautery to expose the vasa.

The vasa in both groups were teased through an 'incision' into a loop with Soonawala forceps and $2 \mathrm{~cm}$ occluded using the long-established Stopes electrocautery technique. ${ }^{1}$ Two of four highly experienced vasectomy surgeons exposed the vasa with the traditional single scalpel 5-7 mm incision using a number 15 or 11 blade. The other two surgeons achieved single wound exposure with the Birtcher 716 reusable electrocoagulation needle powered by the ConMed Hyfrector used for vas occlusion.

Information about the sequelae of the operation was obtained by a self-administered precoded questionnaire mailed, together with prepaid reply envelopes, to participants at 4 and 14 weeks postoperatively.

\section{Methodology}

All clients booked for vasectomies from January to June 1999 with the participating surgeons in London were informed that a trial of the two vas exposure techniques was underway but that the method of occluding the vas was the standard Marie Stopes procedure. Two of the surgeons only carried out the no-scalpel operation while the others only carried out surgery using the scalpel. Those men agreeing to take part in the trial were randomly allocated to a surgeon as far as was possible given their date preferences. A nurse explained the study, procedure and purpose and an appropriate vasectomy trial consent form was signed on the day of the operation.

In the 6-month period from January to June 1999, 325 men volunteered for the trial. Replies to the first questionnaire were received from 275 men, an overall response rate of $84.6 \%$. Of those responding, 165 underwent the no-scalpel procedure and 110 the standard Stopes vasectomy. The second questionnaire elicited 120 responses $(37 \%$ of the original sample and $44 \%$ of the 275 responding to the first questionnaire) of which 66 were from no-scalpel clients and 54 from scalpel clients. Although this response rate is much lower than for the first questionnaire it compares well with other mailed questionnaire studies of the general population. The results were analysed using CIA, the Confident Interval Analysis package developed in conjunction with the British Medical Journal.

\section{Participants}

In order to make comparisons between the two groups the medical records of 100 men from each group were randomly chosen by time within the research period. Data provided from this source comprised age, social class, family size, presence of pre-existing medical problems and whether or not the operation was paid for by the National Health Service (NHS) under an Agency Agreement. A total of 38\% (95\% CI 28.54-8.3) of the noscalpel patients were NHS clients compared to $45 \%(95 \%$ CI 35.0-55.3) of the scalpel group (two in the latter group were unknown)

Table 1 shows the year of birth of the two groups. The results show that an identical number of men in each group were born after 1970 and between 1965 and 1969. In fact there is very little difference between the groups in terms of age group except for the very few men who were born before 1950. Table 2 shows the social class of respondents using the usual market research classification which is based on the Registrar General's five-class designation with the top two and bottom classes merged and the middle one divided into a manual/non-manual divide.

The results for social class (Table 2) show the no- 
Table 1 Year of birth of the two samples ${ }^{\mathrm{a}}$

\begin{tabular}{lll}
\hline Birth year & Non-scalpel & Scalpel \\
\hline After 1970 & 3 & 3 \\
$1965-1969$ & 20 & 20 \\
$1960-1964$ & 30 & 33 \\
$1955-1959$ & 32 & 22 \\
$1950-1954$ & 9 & 12 \\
Before 1950 & 5 & 10 \\
Not stated & 1 & 100 \\
Total & 100 & \\
\hline
\end{tabular}

aThe figures given are both numbers and percentages.

Table 2 Social class of the two samples $\mathrm{a}$

\begin{tabular}{lll}
\hline Social class & Non-scalpel & Scalpel \\
\hline AB & 42 & 28 \\
C1 & 20 & 42 \\
C2 & 23 & 26 \\
DE & 10 & 3 \\
NK & 5 & 1 \\
\hline
\end{tabular}

aThe figures given are both numbers and percentages.

scalpel men had a higher proportion of ABs (professional and managerial). Over two in five $(42 \%)$ of the no-scalpel group were in the highest category compared to less than three in ten $(28 \%)$ of the scalpel group (observed difference $14 \%$ with $95 \%$ CI $-0.9 \%-2.7 \%$ ). However, the no-scalpel group also had more DEs (semiskilled and unskilled). The differences are not statistically significant. The fact that the no-scalpel group was of slightly higher status might be because there were fewer NHS patients.

The subsamples were roughly comparable for family size. In the no-scalpel group, 57 men had two children with an average of 2.14 children in total. In the scalpel group, 59 men had two children with an average of 2.44 in total. There were no reported significant medical problems in either group.

Overall, there was little difference between the groups; consequently any reported differences are likely to be due to differences in the method of operation.

\section{Results}

Participant observation revealed the ENSV procedure, using what the manufacturers of the Hyfrecator describe as 'electro-desiccation' to expose the vas, proved to be a virtually bloodless, simpler and marginally quicker $(<4$ minutes) than the standard Stopes scalpel technique (4-5 minutes). Initial experience revealed that greater care was needed to ensure adequate anaesthesia of the scrotal skin, since diathermy appears to be more painful than a scalpel incision. There was also a need to avoid burning too deeply on making the initial 'incision' because of potential damage to the deferens vessels and a consequent arterial bleed. Closure of the skin wound by fulgurating the edges (but avoiding contact with the unanaesthetised posterior wall of the scrotum) was found to contract the adjacent dartus muscles and effectively closing the wound in most cases.

At 4 weeks post-operation, $43(39 \%)$ of the scalpel group reported problems compared to 54 (33\%) of the ENSV group (6\% difference with $95 \%$ CI $-18 \%-5 \%$ ), which is not statistically significant. Table 3 quantifies the problems reported 4 weeks postoperatively.

Slightly more no-scalpel clients $(26 \%$ vs $21 \%)$ complained of prolonged mild or moderate postoperative pain (5\% difference with $95 \%$ CI $-5 \%-15 \%)$. The essentially bloodless nature of the ENSV procedure is reflected in the recall of postoperative bleeding from the wound of the respondents. Only $8.5 \%$ of the ENSV group found the wound slow to heal compared to $14.5 \%$ of the scalpel group (6\% difference with $95 \%$ CI $-2 \%-14 \%)$. The other areas analysed showed less difference between the groups. There were no reported haematomas or other significant complications in either group.

According to the second mailing at 14 weeks, slightly over two-thirds (37/54) of the scalpel respondents reported bleeding from the wound after leaving the clinic, compared to one-third (22/66) of the ENSV respondents. Although the wound was quicker to heal without the scalpel, and continued bruising slightly less, excessive bruising $(6.5 \%)$ when it did occur was twice as likely with the ENSV procedure (Table 4).

Table 5 shows the speed of return to intercourse. The results show that $52(31.5 \%)$ of the ENSV group returned to intercourse within the first week as compared with 28 $(25.5 \%)$ in the scalpel group $(6 \%$ difference with $95 \%$ CI $-5 \%-17 \%)$. Around three-quarters of both samples had resumed coitus within 2 weeks. As might be expected, those men with problems were slower to resume sexual activity. However, 7/10 of those who had problems after the ENSV operation and 6/10 of those having the scalpel procedure reported having intercourse within 2 weeks.

An important issue for many men, particularly those that are self-employed, is whether the operation will keep them off work with the resultant loss of earnings. At Marie Stopes clinics operations are scheduled on a Friday in order to give clients the weekend to recover, and the vast majority of clients find this sufficient. On average the ENSV clients returned to work in 2.5 days compared to 2.6 days for those having the scalpel procedure. This small difference could easily have occurred by chance and is not a clinically important difference. However, for those experiencing complications, the return to work for the ENSV group was on average a day earlier (3.5 cf 4.5 days). This difference is statistically significant $(\mathrm{p}<0.001)$ (Chisquared test). One in nine men in both groups did not return to work for 7 days or more. However, this was not always because of the operation since one-third reported that the time off was planned. Of those responding to the second mailing, 52\% reported that the ENSV procedure was better than expected as compared to $30 \%$ of the scalpel clients.

Table 3 Nature of the problems experienced according to the method of vas exposure

\begin{tabular}{|c|c|c|c|c|c|c|c|c|c|c|c|c|}
\hline & \multicolumn{2}{|c|}{ Continued pain } & \multicolumn{2}{|c|}{$\begin{array}{l}\text { Wound slow } \\
\text { to heal }\end{array}$} & \multicolumn{2}{|c|}{$\begin{array}{l}\text { Continued } \\
\text { bruising }\end{array}$} & \multicolumn{2}{|c|}{ GP visit } & \multicolumn{2}{|c|}{ Infection } & \multicolumn{2}{|c|}{ Antibiotics } \\
\hline & $\mathrm{n}$ & $\%$ & $\mathrm{n}$ & $\%$ & $\mathrm{n}$ & $\%$ & $\mathrm{n}$ & $\%$ & $\mathrm{n}$ & $\%$ & $\mathrm{n}$ & $\%$ \\
\hline No-scalpel & 43 & 26.0 & 14 & 8.5 & 31 & 18.8 & 18 & 10.9 & 12 & 7.3 & 12 & 7.21 \\
\hline Scalpel & 23 & 20.9 & 16 & 14.5 & 23 & 20.9 & 14 & 12.7 & 6 & 5.5 & 7 & 6.36 \\
\hline Total & 66 & 24.0 & 30 & 10.9 & 54 & 19.6 & 32 & 11.6 & 18 & 6.5 & 19 & 6.9 \\
\hline
\end{tabular}

GP, general practitioner. 


\begin{tabular}{|c|c|c|c|c|c|c|}
\hline & \multicolumn{2}{|c|}{ Mild } & \multicolumn{2}{|c|}{ Moderate } & \multicolumn{2}{|c|}{ Excessive } \\
\hline & $\mathrm{n}$ & $\%$ & $\mathrm{n}$ & $\%$ & $\mathrm{n}$ & $\%$ \\
\hline \multicolumn{7}{|l|}{ Pain } \\
\hline No-scalpel & 16 & 9.6 & 21 & 12.7 & 6 & 3.6 \\
\hline Scalpel & 6 & 5.4 & 12 & 10.9 & 5 & 4.5 \\
\hline Total & 22 & 8.0 & 33 & 12.0 & 11 & 4.0 \\
\hline \multicolumn{7}{|l|}{ Bruising } \\
\hline No-scalpel & 8 & 4.8 & 11 & 6.7 & 12 & 7.2 \\
\hline Scalpel & 6 & 3.6 & 11 & 11.0 & 6 & 3.6 \\
\hline Total & 14 & 4.6 & 22 & 8.0 & 18 & 6.5 \\
\hline
\end{tabular}

aBased on percentage of total sample.

Table 5 Speed of return to intercourse ${ }^{a}$

\begin{tabular}{|c|c|c|c|c|c|c|}
\hline & \multicolumn{2}{|c|}{$<1$ week } & \multicolumn{2}{|c|}{$1-2$ weeks } & \multicolumn{2}{|c|}{$>2$ weeks } \\
\hline & $\mathrm{n}$ & $\%$ & $\mathrm{n}$ & $\%$ & $\mathrm{n}$ & $\%$ \\
\hline \multicolumn{7}{|c|}{ Those with problems } \\
\hline No-scalpel & 15 & 27.8 & 22 & 40.7 & 17 & 31.5 \\
\hline Scalpel & 7 & 16.2 & 18 & 41.9 & 18 & 41.9 \\
\hline \multicolumn{7}{|l|}{ No problems } \\
\hline No-scalpel & 37 & 33.3 & 54 & 48.6 & 20 & 18.0 \\
\hline Scalpel & 21 & 31.3 & 34 & 50.7 & 12 & 17.9 \\
\hline \multicolumn{7}{|l|}{ Total sample } \\
\hline No-scalpel & 52 & 31.5 & 76 & 46.0 & 37 & 22.4 \\
\hline Scalpel & 28 & 25.5 & 52 & 47.3 & 30 & 27.3 \\
\hline Total & 80 & 29.1 & 128 & 46.5 & 67 & 24.4 \\
\hline
\end{tabular}

aPercentages based on the number of men experiencing problems.

\section{Discussion}

The no-scalpel vasectomy (NSV) technique was developed in China by $\mathrm{Dr}$ Li Shungiang of the Chongqing Family Planning Scientific Research Institute in Sichuan Province. Since 1974, over 9 million vasectomies using this technique have been performed. ${ }^{2}$ The procedure uses a clamp to immobilise the vasa just under the skin and a sharp mosquito forceps-like instrument to make a single puncture in the scrotal wall instead of an incision with a scalpel. Comparative studies indicate that the procedure causes less pain, and fewer bleeding and infection problems. ${ }^{3}$ The $\mathrm{Li}$ NSV technique is used in many Marie Stopes family planning clinics in Africa and Asia. The trial of electrocautery rather than a scalpel or the Li instruments to expose the vasa was a logical extension of this experience.

The ENSV proved to be a simple, quick and virtually bloodless procedure requiring just two Soonawala forceps. Although early experience indicated extra care was required to infiltrate the dermis to avoid acute discomfort associated with diathermy of the skin, the peri- and postoperative pain and discomfort for the scalpel and noscalpel technique were comparable. In contrast to the findings of Skiver et al. that the scalpel and no-scalpel procedures had comparable pain and discomfort levels, the ENSV clients reported a slightly higher incidence of mild and moderate postoperative pain, but excessive pain was marginally less. ${ }^{4}$ The return to sexual activity was comparable but for those that did experience postoperative problems the ENSV clients resumed work a day earlier, suggesting their symptoms were of a milder nature.

Operative experience using lignocaine local anaesthesia with or without adrenaline indicates the ENSV procedure is usually a bloodless procedure unless a small vessel is inadvertently bisected as a result of overly aggressive diathermy. The finding that over two-thirds of ENSV clients reported experiencing no postoperative blood loss as compared to just under one-third for the scalpel procedure reflect this experience; although twice as many ENSV clients reported excessive bruising (7.2\%) for which no explanation has been identified. The complication and convalescent experiences for the two groups were comparable.

\section{Conclusions}

The ENSV procedure is a simple, quick, no-touch procedure easily taught and mastered, which is semibloodless requiring only two instruments with minimal complications. Further studies are required to confirm definitely that ENSV is a more acceptable alternative to the scalpel method, however from current evidence ENSV would appear to be suitable for mass application in locations where electricity is available.

\section{Acknowledgements}

The authors wish to thank Dr M Klinger, Dr R Hawkins, Mr N Senthil, Alison Peters, Julie Clark, Helen Axby and Wendy Lutner for their assistance.

\section{Statements on funding and competing interests}

Funding. None identified.

Competing interests. Dr Black is Chief Executive of Marie Stopes International. Professor Francome has co-operated with Marie Stopes International on various surveys and reports.

References

1 Black T, Francome C. The evolution of the Marie Stopes electrocautery no-scalpel vasectomy procedure. J Fam Plann Reprod Health Care 2002: 28: 137-138.

2 Population Report (D). Vasectomy: new opportunities, No. 5, 1992; 3-6.

3 Family Health International. Final report: a comparative study of the no-scalpel and the standard incision method of vasectomy in five countries. Research Triangle Park, NC: Family Health International, 1996.

4 Skriver M, Skovsgaard F, Miskowiak J. Conventional or $\mathrm{Li}$ vasectomy; a questionnaire study. Br J Urol 1997; 79: 596-598. 